\title{
Healthcare Resource Use, Costs, and Disease Progression Associated with Diabetic Nephropathy in Adults with Type 2 Diabetes: A Retrospective Observational Study
}

\author{
Zhou Zhou • Paresh Chaudhari - Hongbo Yang · Anna P. Fang • \\ Jing Zhao • Ernest H. Law · Eric Q. Wu • Ruixuan Jiang • \\ Raafat Seifeldin
}

Received: January 27, 2017 / Published online: March 30, 2017

(c) The Author(s) 2017. This article is an open access publication

\section{ABSTRACT}

Introduction: Diabetic nephropathy (DN) is a progressive kidney disease resulting as a complication of diabetes mellitus. This study evaluated the disease progression and economic burden of DN among commercially insured patients with type 2 diabetes in the USA.

Methods: The research design was a retrospective observational study based on healthcare claims data. The Truven MarketScan Databases (2004-2014) were queried for adults with type 2

A synopsis of the current research was presented at the 52nd European Association for the Study of Diabetes annual meeting, which took place in Munich, Germany during September 12-16, 2016.

Enhanced content To view enhanced content for this article go to http://www.medengine.com/Redeem/4508 F06042A677F0.

Electronic supplementary material The online version of this article (doi:10.1007/s13300-017-0256-5) contains supplementary material, which is available to authorized users.

Z. Zhou $(\bowtie) \cdot$ H. Yang · A. P. Fang · J. Zhao .

E. Q. Wu

Analysis Group, Inc., 111 Huntington Ave, Floor 14,

Boston, MA, USA

e-mail: zhou.zhou@analysisgroup.com

P. Chaudhari

Formerly of Takeda Pharmaceuticals, Inc., One

Takeda Parkway, Deerfield, IL, USA diabetes with at least one urine albumin test (index, randomly selected) after diagnosis and at least one test after the index. On the basis of the index test, patients were classified into normoalbuminuria, microalbuminuria, or macroalbuminuria groups. Nephropathy-related treatment use was measured in the 6 months after the index, disease progression was assessed from the index to the end of data availability, and annual all-cause and nephropathy-related costs and healthcare resource use (HRU) were assessed up to 2 years from the index. Outcomes were compared between any two groups, controlling for baseline demographics.

Results: A total of 23,235 patients were identified and classified into normoalbuminuria $(N=18,409)$, microalbuminuria $(N=3863)$, or macroalbuminuria $(N=963)$ groups. Patients with albuminuria were more likely to be older, male, and have a higher burden of baseline comorbidities and HRU. Within 6 months following the index, 12-20\% of patients with albuminuria were not treated with any relevant recommended treatment. Compared to the

\section{E. H. Law · R. Jiang}

Center for Pharmacoepidemiology and

Pharmacoeconomic Research, University of Illinois at Chicago, Chicago, IL, USA

R. Seifeldin

Takeda Pharmaceuticals, Inc., One Takeda Parkway, Deerfield, IL, USA 
normoalbuminuria group, patients with macroalbuminuria had a significantly greater risk of disease progression (hazard ratio $[\mathrm{HR}]=1.44)$, and both albuminuria groups were more likely to require dialysis $(\mathrm{HR}=4.23$ and 40.14 for micro- and macroalbuminuria, respectively; all $p<0.05)$. Annual all-cause (2016 US dollars, $\$ 3580$ and $\$ 12,830$ higher for micro- and macroalbuminuria vs. normoalbuminuria, respectively) and nephropathy-related (\$362 and \$3716) costs increased significantly with increasing nephropathy severity, consistent with the trend in increased HRU.

Conclusions: Diabetic nephropathy may be undertreated or inappropriately treated. It was also associated with significantly higher costs, HRU, and risk of disease progression among commercially insured patients with type 2 diabetes in the USA.

Funding: Takeda Development Center Americas, Inc.

Keywords: Albuminuria; Heath care costs; Microalbuminuria; Type 2 diabetes

\section{INTRODUCTION}

Diabetic nephropathy (DN) is a progressive kidney disease caused by damage to the small blood vessels in the kidney's glomeruli [1], resulting as a complication of diabetes mellitus (DM) [2]. DN affects up to $40 \%$ of patients with diabetes [3], with a total estimated prevalence of approximately 6.9 million people in the USA [4]. Diabetic nephropathy is a leading cause of end-stage renal disease (ESRD) and greatly increases the risk of allcause mortality, cardiovascular-related morbidity and mortality, and kidney failure among patients with diabetes $[5,6]$. This disease is usually detected by the presence of albuminuria, which is identified on the basis of urine albumin laboratory tests $[3,7,8]$, e.g., urinary albumin excretion rate or albumin-to-creatinine ratio (ACR).

Treatment strategies for patients with mild to severe $\mathrm{DN}$ aim to prevent further renal function decline and delay disease progression via glycemic and blood pressure control, and through inhibition of the renin-angiotensin-aldosterone system (RAAS) [3, 7]. Treatment regimens commonly include RAAS-blocking agents such as angiotensin-converting enzyme (ACE) inhibitors or angiotensin II receptor blockers (ARB). However, these therapies are considered to be inadequate for the treatment of diabetic nephropathy, as they do not reverse the disease $[9,10]$. Treatment combinations, such as dual-RAAS blockade with ACE inhibitors and ARBs, have been considered, but they are associated with adverse events (e.g., hyperkalemia and acute kidney injury); thus, these treatment combinations are not recommended, and are contraindicated in patients with renal impairment $[8,11]$. Finally, even with treatment with currently available therapies, many patients with diabetic nephropathy nevertheless progress to ESRD, requiring dialysis while kidney transplant is considered [3].

As a result of the increased morbidity and mortality associated with diabetic nephropathy, patients with uncontrolled disease face severe economic burden, with estimated average annual healthcare costs ranging from $\$ 8000$ to $\$ 43,000$ [12]. Patients with diabetic nephropathy, especially those with severe stages of nephropathy such as chronic kidney disease and ESRD [12-15], incur higher economic burden compared to those without renal impairment [16-18]. In addition, patients who progress to severe stages of kidney disease experience higher rates of all-cause hospitalization and healthcare resource utilization (HRU) [13], and patients' incremental costs associated with the progression of chronic kidney disease are significantly higher than those of patients without renal impairment $[12,15]$.

Several prior studies have assessed the economic burden of diabetic nephropathy using various definitions of the condition. Nichols et al. assessed the incremental healthcare costs associated with progression to microalbuminuria, macroalbuminuria, and ESRD among patients enrolled in a US region-specific health maintenance organization with both type 2 diabetes mellitus (T2DM) and hypertension [18], which may not adequately represent the overall US patient population with DN. Further, 
medical costs were estimated on the basis of the multiplicative product of "standard" visit costs and frequency of healthcare visits, rather than the observed reimbursement costs. Although there has been some evidence published on UK patients [19], there is currently limited US-specific information on disease progression and the economic burden associated with microalbuminuria or macroalbuminuria among patients with T2DM.

To address this knowledge gap, the current study evaluated real-world disease progression and the associated economic burden, including all-cause and nephropathy-related HRU and costs, among commercially insured USA-based patients with type 2 diabetes and diabetic nephropathy.

\section{METHODS}

\section{Data Source}

The data for this study were derived from the Truven Health Analytics MarketScan ${ }^{\circledR}$ Databases (Jan 1, 2004-Dec 31, 2014). The MarketScan Commercial and Medicare Supplemental claims databases represent approximately 25 million individuals (employees and their dependents, as well as Medicare-eligible retirees) annually covered by over 130 health plans and self-insured employers. The databases contain patient demographics, enrollment history, claims for inpatient and outpatient medical services, and claims for prescription pharmaceuticals. The MarketScan Lab Database contains laboratory test results for a subset of the individuals (approximately 1 million patients) covered in the Commercial and Medicare Supplemental databases, capturing lab tests ordered in office-based practices.

This article does not contain any new studies with human or animal subjects performed by any of the authors. All patient data were de-identified and complied with the patient confidentiality requirements of the Health Insurance Portability and Accountability Act. No ethical approval was required.

\section{Study Population and Subgroups}

The study population included patients with type 2 diabetes who were at least 18 years old and had at least two urine albumin tests (24-h collection or ACR after the first observed type 2 diabetes diagnosis [International Classification of Diseases, 9th revision, Clinical Modification (ICD-9-CM) codes 250.x0 or 250.x2)]. From this population, a subset of patients with at least one eligible urine albumin test meeting the following criteria were identified: (1) at least one additional urine albumin test following the test date; and (2) continuous enrollment in a health plan for at least 12 months before the test date and at least 6 months following the test date and through at least one subsequent urine albumin test. Patients with ESRD (ICD-9-CM codes 403.x1, 404.x2, 404.x3, 585.5, or 585.6) during the 12 -month period prior to the test date were excluded.

For each patient, the presence of microalbuminuria or macroalbuminuria was evaluated on the basis of a randomly selected eligible urine albumin test (index test) and used to classify the patient into one of three groups: (1) the normoalbuminuria group (urinary albumin excretion of less than $30 \mathrm{mg} / 24 \mathrm{~h}$ or an ACR of less than $30 \mu \mathrm{g} / \mathrm{mg}$ ); (2) the microalbuminuria group (urinary albumin excretion of $30-300 \mathrm{mg} / 24 \mathrm{~h}$ or an ACR of $30-300 \mu \mathrm{g} / \mathrm{mg}$ ); and (3) the macroalbuminuria group (urinary albumin excretion of greater than $300 \mathrm{mg} / 24 \mathrm{~h}$ or an ACR of greater than $300 \mu \mathrm{g} / \mathrm{mg}$ ). The date of the index test was defined as the index date.

\section{Study Measures and Outcomes}

Patient demographics, disease characteristics, baseline nephropathy- and diabetes-related treatment use, and all-cause HRU and costs were measured during the 12 -month period prior to the index date (baseline). The overall comorbidity burden during the baseline period was measured using the Charlson comorbidity index (CCI) [20].

Nephropathy-related treatment use was assessed in the 6 months following the index date, and included ACE inhibitors, ARBs, 
diuretics, calcium channel blockers, and other antihypertensive agents, consistent with a prior publication [7]. Disease progression was assessed from the index date until the end of continuous eligibility, end of data availability, or inpatient mortality, whichever came first. Data captured included time to progression to a more severe stage of diabetic nephropathy and time to dialysis/hemodialysis. For the normoalbuminuria group, disease progression was defined as the presence of a urine albumin test indicating microalbuminuria or macroalbuminuria, a diagnosis of ESRD, or a dialysis or renal transplantation procedure. For the microalbuminuria group, disease progression was defined as the presence of a urine albumin test indicating macroalbuminuria, a diagnosis of ESRD, or a dialysis or renal transplantation procedure. For the macroalbuminuria group, disease progression was defined as the presence of a diagnosis of ESRD or a dialysis or renal transplantation procedure.

HRU and healthcare costs were assessed from the index date until 2 years from the index date, the end of continuous eligibility, end of data availability, or patient mortality, whichever occurred first. HRU included all-cause and nephropathy-related inpatient, emergency room (ER), outpatient, and other medical visits. Healthcare costs were evaluated from a thirdparty payer perspective and included all-cause and nephropathy-related healthcare costs resulting from medical services (inpatient, ER, outpatient, and other medical services) and pharmacy prescriptions. Nephropathy-related HRU and costs were defined as medical services and costs associated with a diagnosis of diabetic nephropathy or kidney disease (ICD-9-CM codes 249.4, 250.4, 583.81, 403.xx, 404.xx, or 585.xx), or a procedure for dialysis/hemodialysis or renal transplantation. Costs were converted to 2016 US dollars using the Consumer Price Index medical care component.

\section{Statistical Analyses and Software}

Baseline patient demographics and clinical characteristics were compared across groups using Wilcoxon rank-sum tests for continuous variables and Chi-square tests for categorical variables. Means and standard deviations were reported. Proportions of patients using nephropathy-related treatments during the 6 months following the index date were compared using multivariable logistic regression models, and adjusted odds ratios (ORs) with $p$ values were reported. Time to progression to a more severe disease stage and time to dialysis/ hemodialysis were evaluated using Kaplan-Meier analyses, and compared between study groups using log-rank tests. Cox proportional hazard models were used to further compare any two study groups with adjustment for baseline patient demographics, and adjusted hazard ratios (HRs) with 95\% confidence intervals (CI) and $p$ values were reported. Incidence rates of each type of healthcare visit per patient per year were described and compared using multivariable Poisson regression models, and the adjusted incidence rate ratios (IRRs) with 95\% CIs and $p$ values were reported. To account for over-dispersion, 95\% CIs and $p$ values were estimated using a nonparametric bootstrap resampling technique with 499 iterations. Annual costs in each cost category were described and compared using Wilcoxon rank-sum tests. Adjusted incremental cost differences were estimated using multivariable generalized linear models. For cost components with more than $5 \%$ zero values, a Tweedie distribution with an exponent of 1.67 was employed [21], and for all other components, a gamma distribution and a log link were used. All multivariable models were adjusted for baseline patient demographics, including age at index date, sex, region of residence, and insurance type. Baseline patient characteristics and outcomes were compared pairwise between any two of the three study groups.

Statistical analyses were performed using SAS, version 9.3 (Cary, NC, USA). A $p$ value of 0.05 or less was considered to be significant.

\section{RESULTS}

\section{Baseline Patient Demographic and Clinical Characteristics}

A total of 23,235 patients met the sample selection criteria and were included in the 
analysis. Of these, 18,409 (79\%) patients were classified into the normoalbuminuria group, 3863 (17\%) into the microalbuminuria group, and 963 (4\%) into the macroalbuminuria group (Fig. 1). Baseline demographics such as age, sex, and residential region were different between the three groups (Table 1); patients with microalbuminuria or macroalbuminuria were slightly older (mean age $[\mathrm{SD}]=54.9$ [10.2] and 56 [10.3] years, respectively) and had more men $(60 \%$ and $63 \%)$ than patients within the normoalbuminuria group (mean age $[\mathrm{SD}]=54.3$ [9.6] years, 53\% male).

Patients with more severe nephropathy displayed exacerbated clinical characteristics and higher HRU and costs at baseline. The time from the first observed type 2 diabetes diagnosis in the database to the index date differed between groups, with the macroalbuminuria group having the longest time since diagnosis (mean $[\mathrm{SD}]=44.0$ [30.5] months), followed by the microalbuminuria (40.5 [27.9] months) and normoalbuminuria (37.8 [26.6] months; $p<0.05$ all comparisons) groups (Table 1). A significantly higher comorbidity burden was also observed in patients with microalbuminuria $(\mathrm{CCI}[\mathrm{SD}]=1.9[1.4]$ ) and macroalbuminuria $(2.5$ [1.7] $)$ compared with the normoalbuminuria group (1.6 [1.1]; all $p<0.05)$. With the exception of outpatient services, where $99 \%$ of patients in each group had at least one visit, patients with diabetic nephropathy experienced significantly higher rates of all-cause HRU during the baseline period compared to those with normoalbuminuria ( $p<0.05$ all comparisons), as well as higher

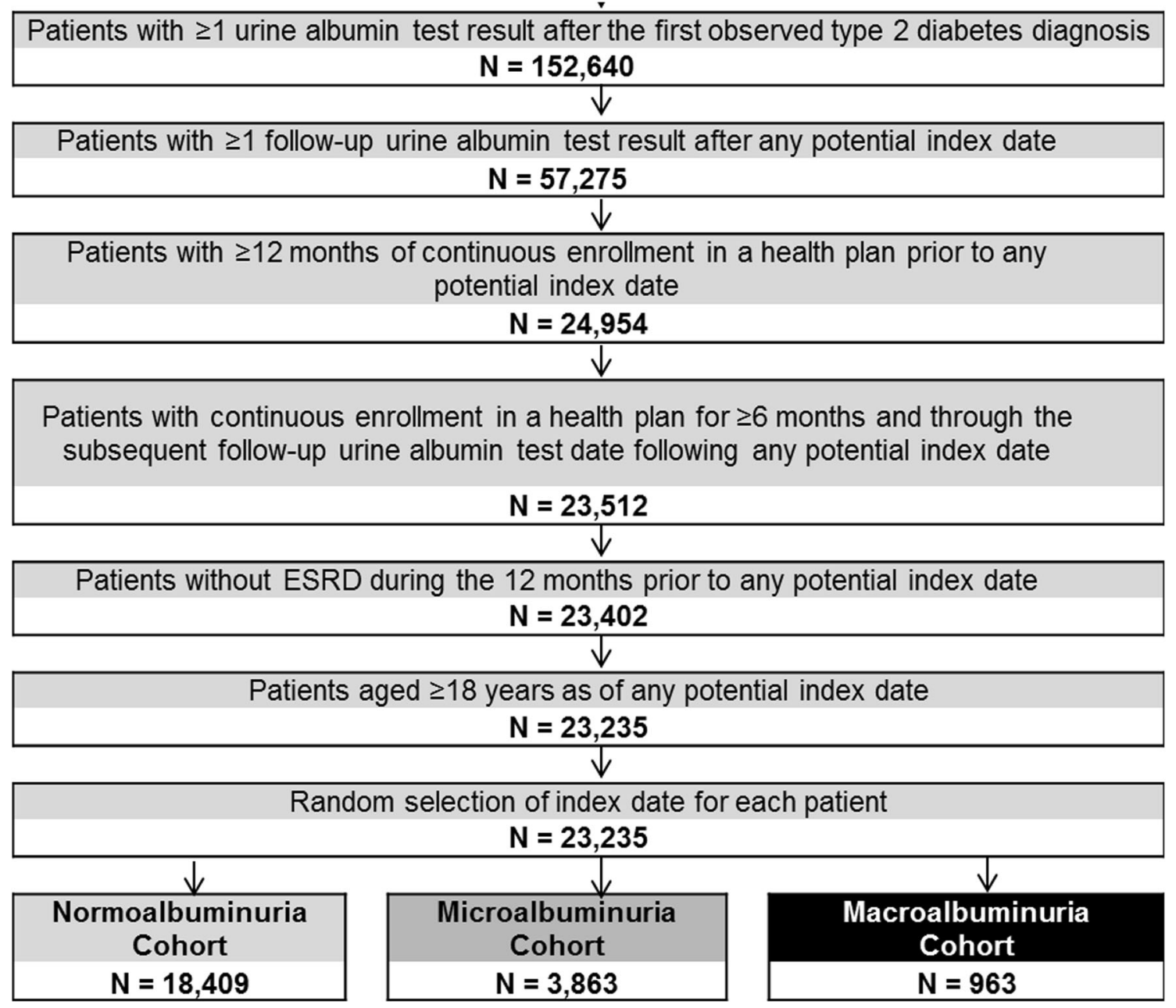

Fig. 1 Selection of study sample. ESRD end-stage renal disease, $N$ number 
Table 1 Patient baseline and clinical characteristics

\begin{tabular}{|c|c|c|c|c|c|c|}
\hline & $\begin{array}{l}\text { Normoalbuminuria } \\
{[\mathrm{A}]} \\
N=18,409\end{array}$ & $\begin{array}{l}\text { Microalbuminuria } \\
{[\mathrm{B}]} \\
N=3863\end{array}$ & $\begin{array}{l}\text { Macroalbuminuria } \\
{[\mathrm{C}]} \\
N=963\end{array}$ & $\begin{array}{l}{[\mathrm{A}] \mathbf{v s .}} \\
{[\mathrm{B}]}\end{array}$ & $\begin{array}{l}{[\mathrm{A}] \text { vs. }} \\
{[\mathrm{C}]}\end{array}$ & $\begin{array}{l}{[B] \text { vs. }} \\
{[\mathrm{C}]}\end{array}$ \\
\hline $\begin{array}{l}\text { Age at index date } \\
\text { (years), mean } \pm S D\end{array}$ & $54.3 \pm 9.6$ & $54.9 \pm 10.2$ & $56.0 \pm 10.3$ & & $\S$ & 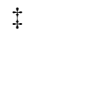 \\
\hline Male, $n(\%)$ & $9836(53 \%)$ & $2310(60 \%)$ & $602(63 \%)$ & $*$ & $\S$ & \\
\hline \multicolumn{7}{|l|}{ Region, $n(\%)$} \\
\hline Northeast & 3577 (19\%) & $686(18 \%)$ & $180(19 \%)$ & * & & \\
\hline North-Central & $4687(25 \%)$ & $1029(27 \%)$ & $289(30 \%)$ & & $\S$ & $\$$ \\
\hline South & $7666(42 \%)$ & $1687(44 \%)$ & $378(39 \%)$ & $*$ & & $\$$ \\
\hline West & $2475(13 \%)$ & $461(12 \%)$ & $116(12 \%)$ & $*$ & & \\
\hline \multicolumn{7}{|c|}{ Insurance plan type, $n(\%)$} \\
\hline $\begin{array}{l}\text { Preferred provider } \\
\text { organization }\end{array}$ & $13,833(75 \%)$ & $2856(74 \%)$ & $711(74 \%)$ & & & \\
\hline $\begin{array}{l}\text { Non-capitated } \\
\text { point-of-service }\end{array}$ & $1421(8 \%)$ & $250(6 \%)$ & $39(4 \%)$ & $*$ & $\S$ & 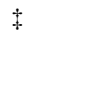 \\
\hline $\begin{array}{l}\text { Exclusive provider } \\
\text { organization }\end{array}$ & $911(5 \%)$ & $191(5 \%)$ & $52(5 \%)$ & & & \\
\hline $\begin{array}{l}\text { Comprehensive } \\
\text { traditional plan }\end{array}$ & $1597(9 \%)$ & $434(11 \%)$ & $136(14 \%)$ & * & $\S$ & 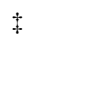 \\
\hline Other plans & $647(4 \%)$ & $132(3 \%)$ & $25(3 \%)$ & & & \\
\hline $\begin{array}{l}\text { Time from first } \\
\text { observed type } 2 \\
\text { diabetes diagnosis in } \\
\text { the database to the } \\
\text { index date (months), } \\
\text { mean } \pm S D\end{array}$ & $37.8 \pm 26.6$ & $40.5 \pm 27.9$ & $44.0 \pm 30.5$ & $*$ & $\S$ & $\$$ \\
\hline \multicolumn{7}{|c|}{ Index urine albumin test characteristics } \\
\hline \multicolumn{7}{|c|}{ 24-h urine albumin (mg) } \\
\hline$n(\%)$ & $208(1 \%)$ & $57(1 \%)$ & $24(2 \%)$ & & & \\
\hline Mean $\pm S D$ & $7.5 \pm 7.1$ & $97.9 \pm 73.5$ & $2289.4 \pm 2044.7$ & $*$ & $\S$ & $\$$ \\
\hline Median $[\min , \max ]$ & $7.2[0.0,29.1]$ & $73.0[30.0,281.0]$ & $\begin{array}{l}1519.5[315.0 \\
7140.2]\end{array}$ & & & \\
\hline \multicolumn{7}{|c|}{ Albumin/creatinine ratio $(\mu \mathrm{g} / \mathrm{mg})$} \\
\hline$n(\%)$ & $18,201(99 \%)$ & $3806(99 \%)$ & $939(98 \%)$ & & & \\
\hline Mean $\pm S D$ & $7.6 \pm 6.5$ & $88.6 \pm 62.7$ & $3439.6 \pm 11,630.7$ & $*$ & $\S$ & $\$$ \\
\hline
\end{tabular}


Table 1 continued

\begin{tabular}{|c|c|c|c|c|c|c|}
\hline & $\begin{array}{l}\text { Normoalbuminuria } \\
{[\mathrm{A}]} \\
N=18,409\end{array}$ & $\begin{array}{l}\text { Microalbuminuria } \\
{[\mathrm{B}]} \\
N=3863\end{array}$ & $\begin{array}{l}\text { Macroalbuminuria } \\
{[\mathrm{C}]} \\
N=963\end{array}$ & $\begin{array}{l}{[\mathrm{A}] \text { vs. }} \\
{[\mathrm{B}]}\end{array}$ & $\begin{array}{l}{[\mathrm{A}] \text { vs. }} \\
{[\mathrm{C}]}\end{array}$ & $\begin{array}{l}{[B] \text { vs. }} \\
{[C]}\end{array}$ \\
\hline Median $[\min , \max ]$ & $6.0[0.0,29.8]$ & $64.5[30.0,297.0]$ & $\begin{array}{c}734.0[300.4, \\
90,000.0]\end{array}$ & & & \\
\hline $\mathrm{CCI}$, mean $\pm \mathrm{SD}$ & $1.6 \pm 1.1$ & $1.9 \pm 1.4$ & $2.5 \pm 1.7$ & $*$ & $\S$ & $\ddagger$ \\
\hline \multicolumn{7}{|c|}{ Disease-specific comorbidities, $n(\%)$} \\
\hline Neuropathy & $2355(13 \%)$ & $618(16 \%)$ & $218(23 \%)$ & $*$ & $\S$ & $\ddagger$ \\
\hline Ischemic heart disease & $2191(12 \%)$ & $602(16 \%)$ & $192(20 \%)$ & $*$ & $\S$ & $\ddagger$ \\
\hline Retinopathy & $2004(11 \%)$ & $593(15 \%)$ & $251(26 \%)$ & $*$ & $\S$ & $\ddagger$ \\
\hline Anemia & $1358(7 \%)$ & $356(9 \%)$ & $155(16 \%)$ & $*$ & $\S$ & $\ddagger$ \\
\hline Depression & $1198(7 \%)$ & $219(6 \%)$ & $51(5 \%)$ & & & \\
\hline $\begin{array}{l}\text { Cerebrovascular } \\
\text { disease }\end{array}$ & $852(5 \%)$ & $255(7 \%)$ & $102(11 \%)$ & $*$ & $\S$ & $\ddagger$ \\
\hline Heart failure & $370(2 \%)$ & $159(4 \%)$ & $60(6 \%)$ & $*$ & $\S$ & $\ddagger$ \\
\hline Ketoacidosis & $150(1 \%)$ & $37(1 \%)$ & $22(2 \%)$ & & $\S$ & $t$ \\
\hline Hyperkalemia & $111(1 \%)$ & $55(1 \%)$ & $23(2 \%)$ & $*$ & $\S$ & + \\
\hline $\begin{array}{l}\text { High parathyroid } \\
\text { hormone level }\end{array}$ & $106(1 \%)$ & $33(1 \%)$ & $22(2 \%)$ & $*$ & $\S$ & $\ddagger$ \\
\hline High phosphorus level & $16(<1 \%)$ & $5(<1 \%)$ & $3(<1 \%)$ & & & \\
\hline \multicolumn{7}{|c|}{ Disease-specific treatments, $n(\%)$} \\
\hline $\begin{array}{l}\text { Nephropathy-related } \\
\text { treatments }\end{array}$ & $12,726(69 \%)$ & $3032(78 \%)$ & $830(86 \%)$ & $*$ & $\S$ & $\ddagger$ \\
\hline ACE inhibitor & $6199(34 \%)$ & $1548(40 \%)$ & $427(44 \%)$ & $*$ & $\S$ & $\ddagger$ \\
\hline Diuretic & $3276(18 \%)$ & $850(22 \%)$ & $339(35 \%)$ & $*$ & $\S$ & $\ddagger$ \\
\hline $\begin{array}{l}\text { Calcium channel } \\
\text { blocker }\end{array}$ & $2330(13 \%)$ & $811(21 \%)$ & $296(31 \%)$ & $*$ & $\S$ & $\ddagger$ \\
\hline $\mathrm{ARB}$ & $2293(12 \%)$ & $654(17 \%)$ & $208(22 \%)$ & $*$ & $\S$ & $\ddagger$ \\
\hline $\begin{array}{l}\text { Other } \\
\text { antihypertensive } \\
\text { agents }\end{array}$ & $4661(25 \%)$ & $1132(29 \%)$ & $369(38 \%)$ & $*$ & $\S$ & $\ddagger$ \\
\hline $\begin{array}{l}\text { Diabetic treatments, } \\
n(\%)\end{array}$ & $15,177(82 \%)$ & $3363(87 \%)$ & $852(88 \%)$ & $*$ & $\S$ & \\
\hline Metformin & $11,548(63 \%)$ & $2532(66 \%)$ & $510(53 \%)$ & $*$ & $\S$ & $\ddagger$ \\
\hline Sulfonylureas & $4293(23 \%)$ & $1125(29 \%)$ & $314(33 \%)$ & $*$ & $\S$ & $\ddagger$ \\
\hline Insulin & $3915(21 \%)$ & $1179(31 \%)$ & $459(48 \%)$ & $*$ & $\S$ & $\ddagger$ \\
\hline
\end{tabular}


Table 1 continued

\begin{tabular}{|c|c|c|c|c|c|c|}
\hline & $\begin{array}{l}\text { Normoalbuminuria } \\
{[\mathrm{A}]} \\
N=18,409\end{array}$ & $\begin{array}{l}\text { Microalbuminuria } \\
{[\mathrm{B}]} \\
N=3863\end{array}$ & $\begin{array}{l}\text { Macroalbuminuria } \\
{[\mathrm{C}]} \\
N=963\end{array}$ & $\begin{array}{l}{[\mathrm{A}] \text { vs. }} \\
{[\mathrm{B}]}\end{array}$ & $\begin{array}{l}{[\mathrm{A}] \mathrm{vs.}} \\
{[\mathrm{C}]}\end{array}$ & $\begin{array}{l}{[\mathrm{B}] \text { vs. }} \\
{[\mathrm{C}]}\end{array}$ \\
\hline DPP4 inhibitor & $1756(10 \%)$ & $438(11 \%)$ & $111(12 \%)$ & * & $\S$ & \\
\hline GLP1-based therapy & $1279(7 \%)$ & $293(8 \%)$ & $72(7 \%)$ & & & \\
\hline SGLT2 inhibitor & $3(<1 \%)$ & $0(0 \%)$ & $0(0 \%)$ & & & \\
\hline $\begin{array}{l}\text { Other antidiabetic } \\
\text { agents }\end{array}$ & $3847(21 \%)$ & $911(24 \%)$ & $259(27 \%)$ & * & $\S$ & $\ddagger$ \\
\hline \multicolumn{7}{|c|}{ All-cause healthcare resource utilization } \\
\hline \multicolumn{7}{|c|}{ Patients with $\geq 1$ visit, $n(\%)$} \\
\hline Inpatient admissions & $1490(8 \%)$ & $453(12 \%)$ & $169(18 \%)$ & * & $\S$ & $\$$ \\
\hline ER services & $4150(23 \%)$ & $984(25 \%)$ & $263(27 \%)$ & * & $\S$ & \\
\hline Outpatient services & $18,226(99 \%)$ & 3817 (99\%) & $952(99 \%)$ & & & \\
\hline Other & $10,073(55 \%)$ & $2186(57 \%)$ & $610(63 \%)$ & * & $\S$ & $\ddagger$ \\
\hline \multicolumn{7}{|c|}{ Number of visits per patient, mean \pm SD } \\
\hline Inpatient admissions & $0.1 \pm 0.4$ & $0.2 \pm 0.5$ & $0.2 \pm 0.6$ & * & $\S$ & $\ddagger$ \\
\hline Inpatient days & $0.5 \pm 2.9$ & $0.8 \pm 3.4$ & $1.4 \pm 4.5$ & * & $\S$ & $\$$ \\
\hline ER services & $0.4 \pm 1.2$ & $0.5 \pm 1.4$ & $0.6 \pm 2.0$ & * & $\S$ & \\
\hline Outpatient services & $12.9 \pm 12.6$ & $12.9 \pm 12.4$ & $14.0 \pm 12.4$ & & $\S$ & 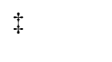 \\
\hline Other & $2.0 \pm 4.1$ & $2.5 \pm 4.9$ & $3.5 \pm 6.7$ & * & $\S$ & 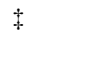 \\
\hline \multicolumn{7}{|c|}{ All-cause healthcare costs (2016 USD), mean \pm SD } \\
\hline Total healthcare costs & $10,602.7 \pm 19,847.4$ & $12,882.6 \pm 25,539.6$ & $15,849.0 \pm 23,728.3$ & * & $\S$ & $\ddagger$ \\
\hline Total medical costs & $7108.4 \pm 18,682.0$ & $9018.6 \pm 24,305.0$ & $11,184.5 \pm 21,995.8$ & * & $\S$ & $\ddagger$ \\
\hline $\begin{array}{l}\text { Total pharmaceutical } \\
\text { costs }\end{array}$ & $3494.3 \pm 4869.1$ & $3864.0 \pm 5130.1$ & $4664.5 \pm 6426.0$ & * & $\$$ & * \\
\hline
\end{tabular}

$A C E$ angiotensin-converting enzyme, $A R B$ angiotensin II receptor blocker, $C C I$ Charlson comorbidity index, DPP4 dipeptidyl peptidase 4, ER emergency room, GLP1 glucagon-like peptide-1, $N$ number, $S D$ standard deviation, SGLT2 sodium-glucose cotransporter 2, USD United States dollars

${ }^{*} p<0.05$ for [A] vs. [B]; ${ }^{\S} p<0.05$ for [A] vs. [C]; ${ }^{\dagger} p<0.05$ for [B] vs. [C]

all-cause healthcare costs $(p<0.05$, all comparisons) (Table 1).

\section{Nephropathy-Related Treatment Use and Disease Progression During the Follow-up Period}

During the 6 months following the index date, nephropathy-related treatments were used by
$68 \%$ with normal urine albumin levels, $81 \%$ of patients with microalbuminuria, and $88 \%$ of patients with macroalbuminuria (Table S1). ACE inhibitors were the most commonly used treatment for nephropathy $(32 \%, 41 \%$, and $43 \%$ for the normoalbuminuria, microalbuminuria, and macroalbuminuria groups, respectively). Both ACE inhibitors and ARBs were used by $3 \%$ of patients with 
microalbuminuria and $6 \%$ of patients with macroalbuminuria.

During the follow-up period, patients with microalbuminuria or macroalbuminuria had a significantly greater risk of progression to a more severe disease stage compared to patients in the normoalbuminuria group (HRs $[\mathrm{CI}]=1.31[1.08,1.60]$ and $1.44[1.21,1.72]$, respectively; both $p<0.05$ ) (Fig. 2 a). Specifically, the 5-year disease progression rates were $15 \%, 19 \%$, and $31 \%$ for the normoalbuminuria, microalbuminuria, and macroalbuminuria groups, respectively. Patients with microalbuminuria and macroalbuminuria were at significantly higher risk of receiving dialysis/ hemodialysis compared to patients with normoalbuminuria (HRs $[\mathrm{CI}]=4.23[2.45,7.30]$ and 40.14 [25.33, 63.60], respectively; both $p<0.001$ ) (Fig. 2b). The 5-year dialysis rates were $0.3 \%$ among patients with normal albumin levels, $2 \%$ among those with microalbuminuria, and $18 \%$ among those with macroalbuminuria.

\section{Annual All-Cause and Nephropathy-Related HRU During the Follow-up Period}

The annual frequency of all-cause healthcare visits rose significantly with the increasing severity of the patient's nephropathy, a trend that was consistent across inpatient, outpatient, $\mathrm{ER}$, and other medical services visits $(p<0.05$ in all pairwise adjusted IRR comparisons) (Table 2 ). The microalbuminuria group experienced significantly higher utilization rates of each type of all-cause medical service in comparison with the normoalbuminuria group, particularly inpatient admissions (adjusted IRR [CI] $=1.51$ $[1.37,1.65] ; p<0.05$ all adjusted IRR comparisons) (Table 2). Furthermore, the macroalbuminuria group experienced particularly heightened average annual incidence rates of all-cause inpatient admissions in comparison with the microalbuminuria and normoalbuminuria groups (adjusted IRRs [CI] of 1.78 [1.50, $2.09]$ and 2.70 [2.34, 3.16], respectively; both $p<0.05)$. A similar trend was observed in healthcare visits related to nephropathy, with an increased magnitude of difference between groups. In comparison with the normoalbuminuria group, the microalbuminuria group had significantly higher incidence rates of nephropathy-related inpatient (adjusted IRR $[\mathrm{CI}]=2.94[2.21,3.88])$, ER $(4.70[3.09,7.76])$, outpatient (3.77 [3.16, 4.48]), and other medical service visits $(6.26[2.43,19.46]$, all $p<0.05)$ (Table 2). Additionally, in comparison with both the microalbuminuria and normoalbuminuria groups, patients with macroalbuminuria had significantly higher incidence rates of nephropathy-related inpatient (adjusted IRRs $[\mathrm{CI}]=5.24[3.91,7.09]$ and $16.53[12.14,21.87]$, respectively), ER $(4.03[2.49,6.46]$ and 18.96 $[11.79,29.88])$, outpatient $(4.83[3.51,6.30]$ and $18.25[13.36,24.86])$, and other medical services visits $(8.54[3.87,19.92]$ and $50.20[16.45$, 174.00]; all $p<0.05$ ) during the follow-up period, after adjusting for baseline patient demographics (Table 2). For both all-cause and nephropathy-related HRU, the differences in adjusted annual HRU rates between the albuminuria and normoalbuminuria groups increased with disease severity, with the macroalbuminuria group exhibiting the highest HRU in all cases.

\section{Annual All-Cause and Nephropathy-Related Healthcare Costs During the Follow-up Period}

Patients with microalbuminuria or macroalbuminuria had significantly higher annual all-cause healthcare costs (2016 US dollars) compared to those in the normoalbuminuria group (mean [SD], normoalbuminuria $=\$ 12,353 \quad[20,082]$, microalbuminuria $=$ $\$ 15,893 \quad[29,874], \quad$ macroalbuminuria $=$ $\$ 25,424[47,844])$, with adjusted cost differences of $\$ 3580$ and $\$ 12,830$ per year, respectively ( $p<0.05$, all comparisons) (Table 3 ). The microalbuminuria group had higher costs than those of the normoalbuminuria group $(p<0.05$, all comparisons), and the macroalbuminuria group had significantly higher adjusted all-cause healthcare costs than either the normoalbuminuria or microalbuminuria groups in all categories (with the exception of 

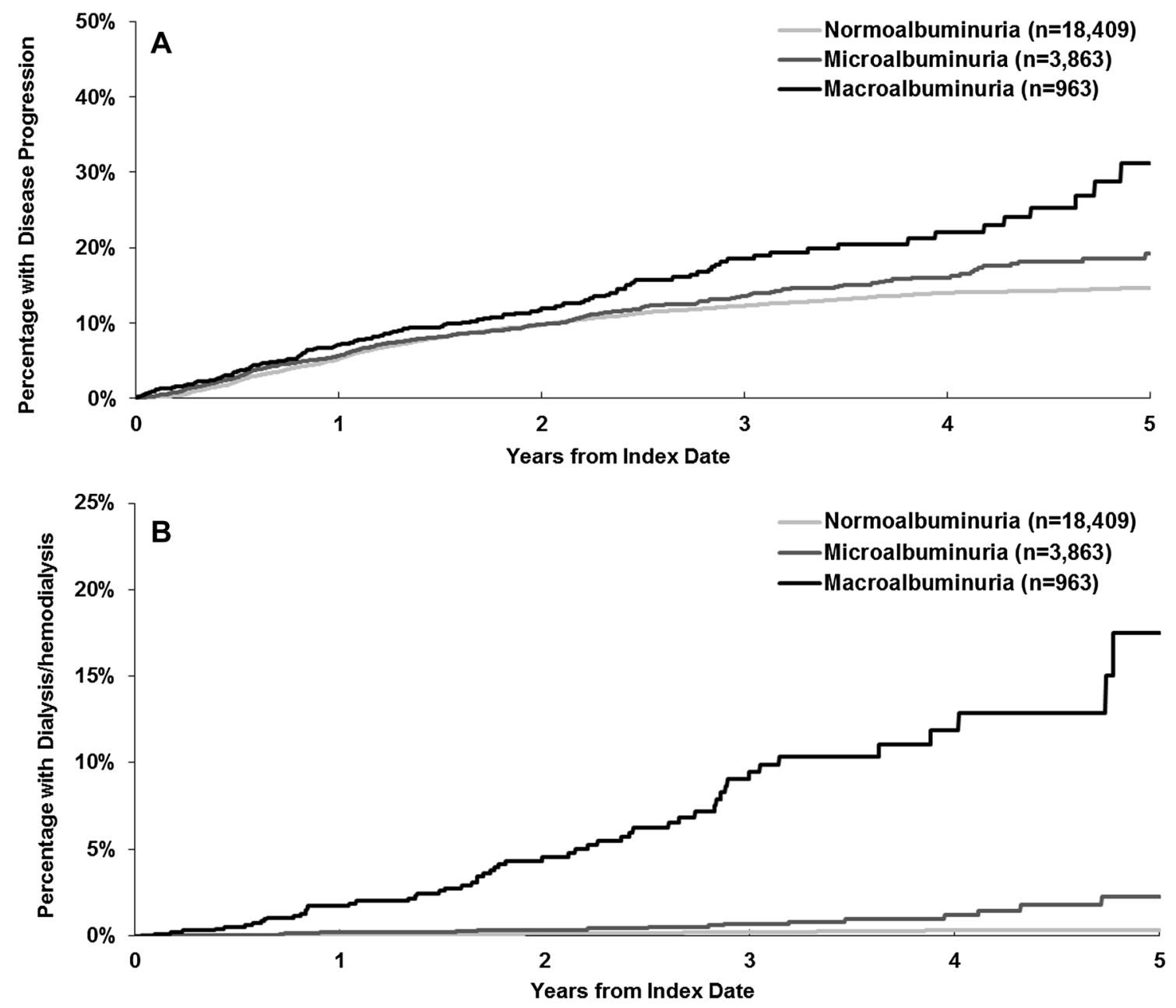

Fig. 2 Comparison of 1-, 3-, and 5-year clinical outcomes between groups. a Rates of disease progression: the median number of follow-up years for the macro-, micro-, and normoalbuminuria groups was $1.81,1.93$, and 1.95 years, respectively. The 1-, 3-, and 5-year progression rates for the macroalbuminuria group were $7.02 \%, 18.51 \%$, and $31.19 \%$, those for the microalbuminuria group were $5.64 \%, 13.49 \%$, and $19.19 \%$, and those for the normoalbuminuria group were $5.26 \%, 12.25 \%$, and $14.68 \%$.

ER visits in comparison with the microalbuminuria group) (Table 3 ). Inpatient admissions costs accounted for $45-51 \%$ of the adjusted cost differences between groups, followed by outpatient (20-24\%) and pharmaceutical costs (10-15\%). A similar trend was observed in adjusted nephropathy-related costs (Table 3 ). Compared to patients in the normoalbuminuria group, patients with microalbuminuria b Comparison of time to dialysis/hemodialysis: the median number of follow-up years for the macro-, micro-, and normoalbuminuria groups was 1.92, 2.06, and 2.13 years, respectively. The 1-, 3-, and 5-year dialysis rates for the macroalbuminuria group were $1.75 \%, 9.46 \%$, and $17.52 \%$, those for the microalbuminuria group were $0.19 \%, 0.71 \%$, and $2.25 \%$, and those for the normoalbuminuria group were $0.02 \%, 0.19 \%$, and $0.33 \%$. $n$ number

and macroalbuminuria incurred higher total nephropathy-related adjusted healthcare costs, with increases of $\$ 362$ and $\$ 3716$, respectively. The nephropathy-related total annual healthcare costs among patients with macroalbuminuria were $\$ 4427$, driven by high inpatient (mean $[\mathrm{SD}]=\$ 2048[10,765]$ ) and outpatient $(\$ 1526[11,515])$ medical costs. 


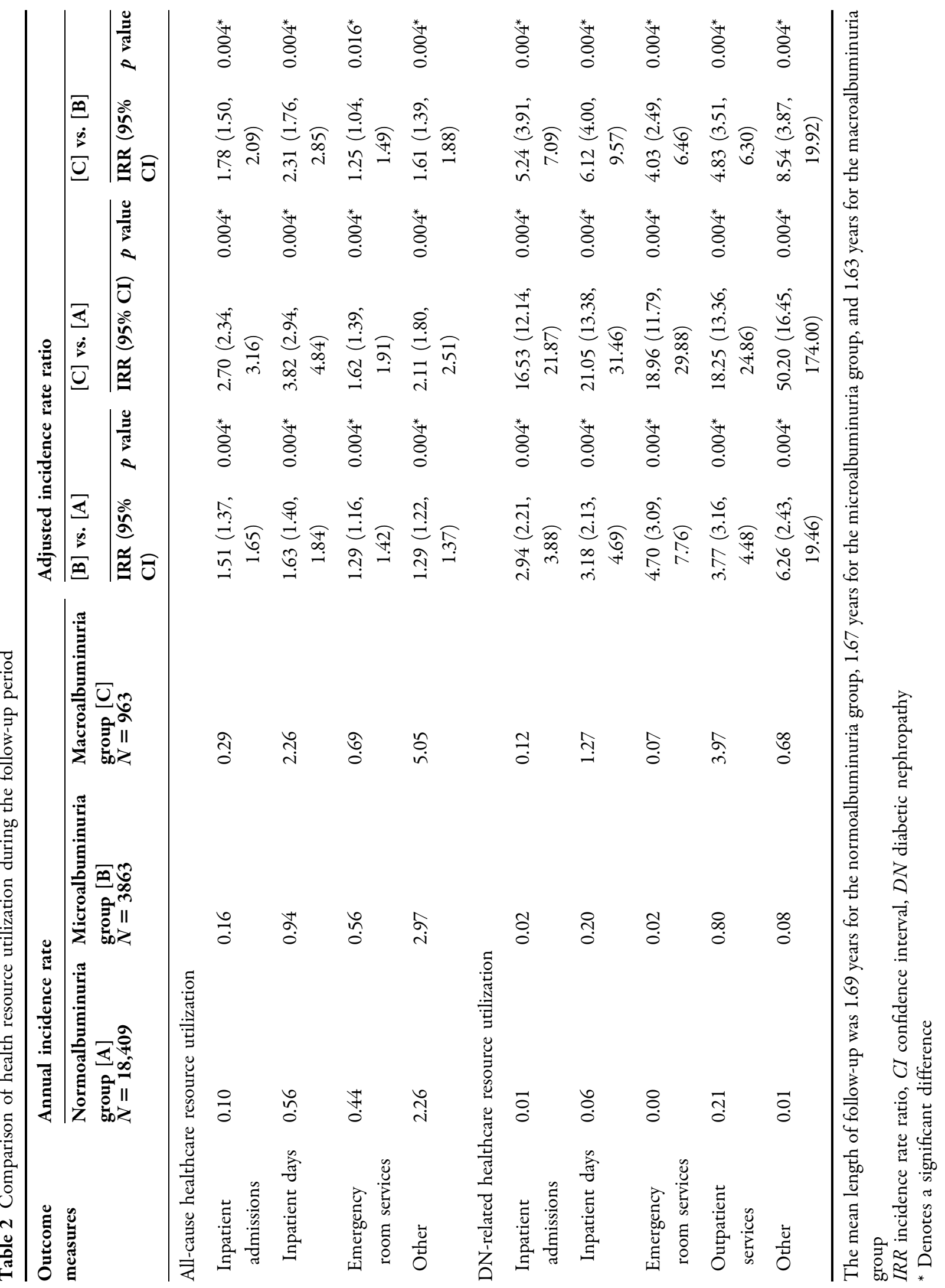




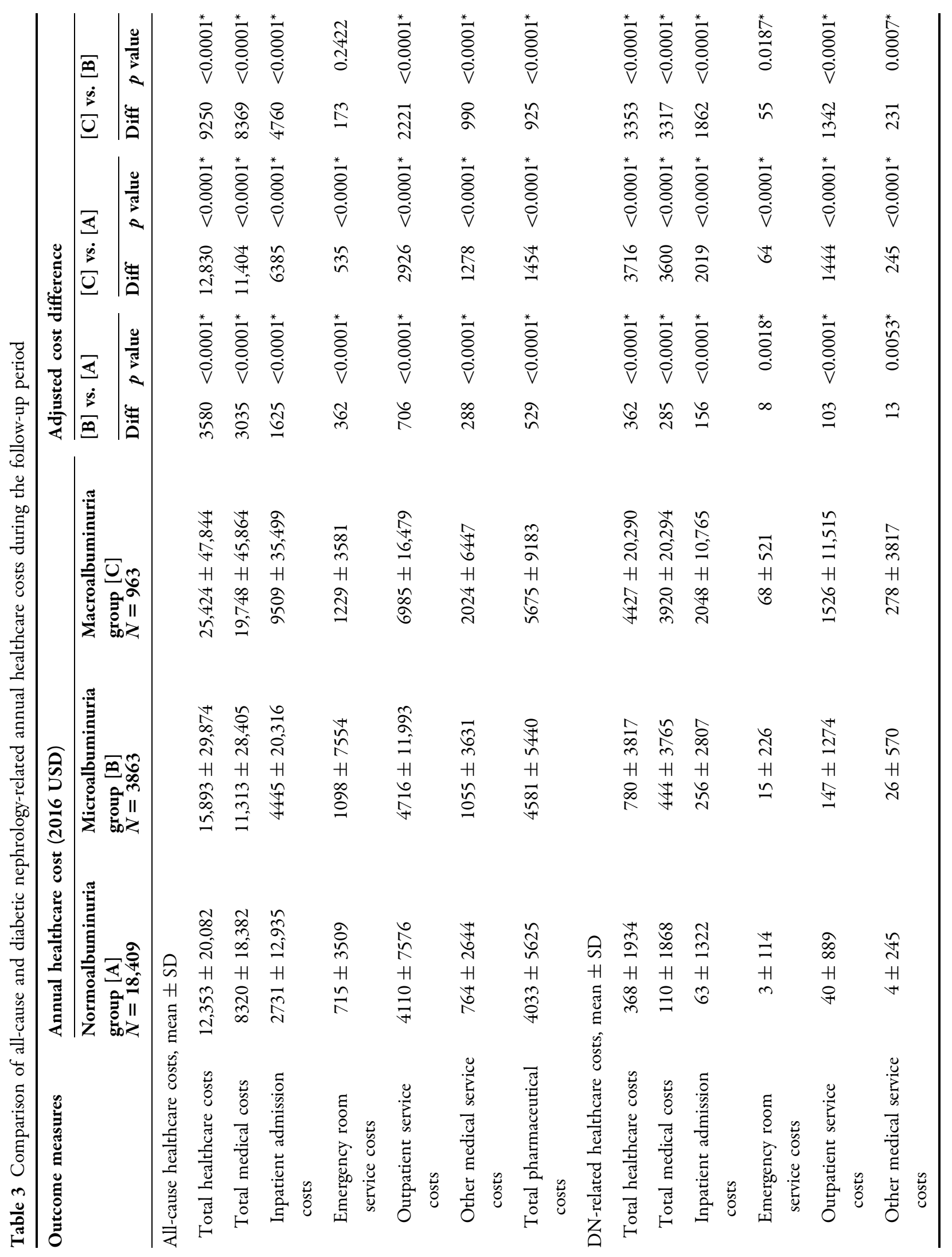




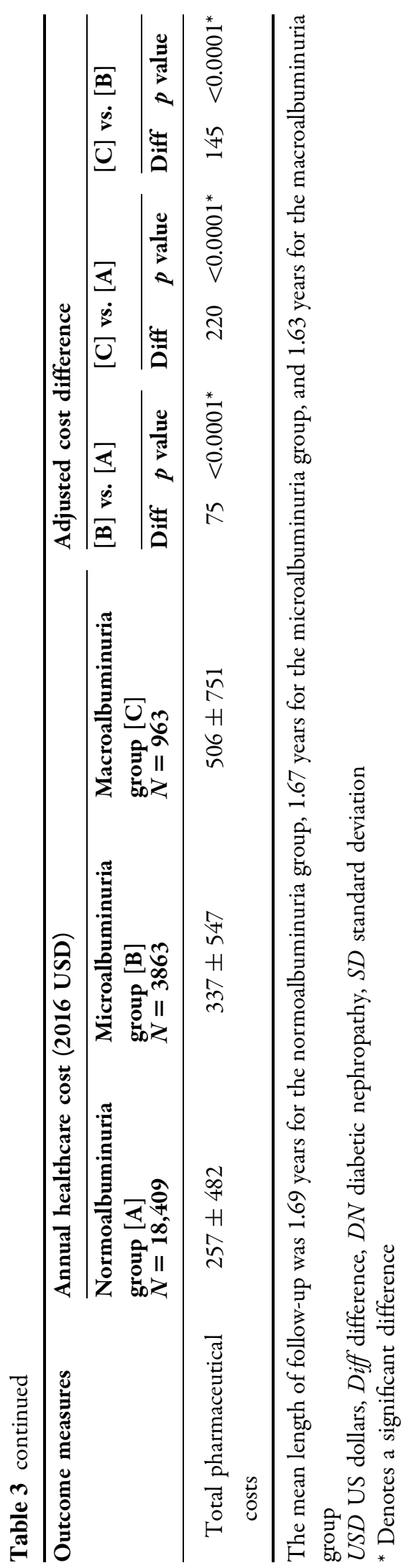

\section{DISCUSSION}

The current study quantifies and underscores the significant risk of disease progression and incremental economic burden associated with microalbuminuria and macroalbuminuria compared with no renal impairment, as well as the progressive nature of such outcomes.

The results suggest that the incremental benefit of treating early-stage diabetic nephropathy may be substantial. A nearly 50\% increased risk of mortality associated with albuminuria has been observed with very low levels not previously believed to be impactful (15-29 mg/24 h), suggesting an expansion of the category of diabetic patients who may benefit from early intervention [22] or screening [23]. Given the significantly higher risk of requiring costly dialysis and renal transplantation procedures in patients with albuminuria, reducing disease progression rates would result in significant cost savings. Accordingly, the development of effective treatments and early intervention strategies could reduce the risk of adverse clinical outcomes and ultimately reduce HRU and costs.

Most importantly, the high disease progression rates observed in this study, despite the availability of treatments of diabetic nephropathy, highlight the inadequacy of current DN treatments and the need for treatments that can effectively delay disease progression. In 2002, Adler et al. estimated DN progression rates in a UK population; progression rates reported in the current study are higher than those reported by Adler et al. in particular among patients with macroalbuminuria [19]. However, our study is not directly comparable to Adler et al.'s study for several reasons. Adler et al. defined disease progression among patients with macroalbuminuria by elevated plasma creatinine or renal replacement therapy [19], and microalbuminuria was defined using the UK Prospective Diabetes Study standards (urinary albumin excretion of $50-299 \mathrm{mg} / 24 \mathrm{~h}$ ) [24]. In the current study, microalbuminuria was defined as $30-300 \mathrm{mg} / 24 \mathrm{~h}$ or an ACR of $30-300 \mu \mathrm{g} / \mathrm{mg}$, and macroalbuminuria was defined on the basis of $24 \mathrm{~h}$ albumin, namely greater than $300 \mathrm{mg} /$ 
$24 \mathrm{~h}$ or ACR of greater than $300 \mu \mathrm{g} / \mathrm{mg}$. Adler et al.'s study also classified the stages of diabetic nephropathy based on two consecutive annual visits while the current study used only one visit to define the stages of DN, which might have resulted in an overestimation of the rates of progression. Despite these differences, the current study provides clinical evidence more applicable to the US population than Adler et al.'s study as it was based on the US population and had more than four times the number of included patients. Among other studies conducted within the USA, disease progression rates are higher than those reported by Adler et al. in the UK, and more consistent with the findings of the present study; the Wisconsin Epidemiologic Study of Diabetic Retinopathy examined rates of disease progression among diabetic patients and reported that during the 15 years following a diagnosis of diabetes, $45.2 \%$ of patients developed either microalbuminuria or macroalbuminuria [5].

Disease progression in diabetic nephropathy has been previously reported to be accompanied by rising medical treatment costs. Nichols et al. found that patients with type 2 diabetes and hypertension who progressed from normal urine albumin levels to microalbuminuria or microalbuminuria to macroalbuminuria incurred higher incremental annual costs of $\$ 2764$ and $\$ 3618$ from pre-progression to post-progression, respectively [18]. However, that study focused on a type 2 diabetes population limited to those with hypertension, which may not be generalizable to the entire diabetic nephropathy population, and costs were imputed using "standard" unit costs for visit types and visits frequencies rather than actual costs incurred. Conversely, the current study included a broadly defined population-any patients with type 2 diabetes-and compared both the frequency of healthcare visits and actual incurred healthcare costs between patients with and without nephropathy, as well as between patients with macroalbuminuria versus microalbuminuria. We found that, consistent with worsening clinical outcomes, patients with microalbuminuria and macroalbuminuria had higher all-cause and nephrology-related HRU and a substantial economic burden. However, the adjusted incremental all-cause cost burdens associated with macroalbuminuria as compared to microalbuminuria (an adjusted difference of $\$ 9250$ ) or patients without albuminuria $(\$ 12,830)$, as well as that of microalbuminuria compared with patients without albuminuria (\$3580), were higher than those found by Nichols et al. This difference may be due to differing levels of disease progression during follow-up between the two studies; only $5 \%$ of patients with macroalbuminuria were observed to progress to ESRD in Nichols et al. [18] (over 6.5 years of follow-up), compared to the estimated 5-year progression rate of $31 \%$ in the current study. Additionally, the current study observed higher annual costs at baseline among the normoalbuminuria, microalbuminuria, and macroalbuminuria groups, which may be indicative of underlying differences in cost calculations compared to that study.

This study also demonstrated that despite abnormal urine albumin test results, many patients are not receiving treatment for diabetic nephropathy-between $12 \%$ and $20 \%$ remained untreated during the 6 months following an abnormal urine albumin test result. However, among those treated, less than half of patients used ACE inhibitors or ARBs, the current treatments recommended by the American Diabetes Association (ADA) for patients with elevated urinary albumin excretion [8]. The results of this study have additional important implications for physicians treating patients with type 2 diabetes as well as for payers as it demonstrates the possible undertreatment and inappropriate treatment of patients with DN. Given the incremental benefit of treating early-stage diabetic nephropathy, both DN diagnosis and appropriate treatment must be timely as they are critical for delaying disease progression and reducing healthcare costs.

This study was subject to certain limitations. Patients were required to be continuously enrolled for at least 6 months after the index date and until the follow-up urine albumin test, which may introduce immortal time bias. However, as the mean follow-up time was similar among groups (1.69 years for the normoalbuminuria group, 1.67 years for the microalbuminuria group, and 1.63 years for the 
macroalbuminuria group) the impact of this bias is expected to be similar across groups; thus, the results would be expected to be qualitatively similar to the results if immortal time bias was completely eliminated. The use of a single urine albumin test to determine increased urinary albumin excretion rates may cause misclassification of disease progression; if the urine albumin was transiently elevated, an underestimation of the difference between groups may have occurred.

Although the database is geographically representative of the USA, the data included only commercially insured patients and those who have commercial insurance in supplement to Medicare. In addition, to evaluate disease progression of diabetic nephropathy, the current study sample was further restricted to the subset of patients who were also in the MarketScan Lab Database and who had two urine albumin tests results. Thus, the results may not be generalizable across all study populations.

The selection of patients with two urine albumin tests for inclusion in the study may bias the study population toward one at high risk for diabetic nephropathy, who requires frequent monitoring of urine albumin levels, thus potentially biasing the study results toward greater progression rates than those of the general type 2 diabetic population. However, because the study results are consistent with a US prospective study, the selection bias is minimized. Furthermore, the limited information available in the database prevented further analysis on important patient characteristics (e.g., diabetes disease duration since the initial diagnosis, race), indirect costs (e.g., lost productivity), and additional important clinical measures (e.g., HbA1c, eGFR).

\section{CONCLUSION}

Microalbuminuria and macroalbuminuria were associated with significantly higher risk of disease progression and substantially increased economic burden among commercially insured patients with type 2 diabetes in the USA. These results highlight the persisting unmet need for effective treatments and early intervention strategies for adult patients with diabetic nephropathy.

\section{ACKNOWLEDGEMENTS}

Sponsorship for this study and article processing charges were funded by Takeda Development Center Americas, Inc. The study sponsor was involved in all stages of the study research, manuscript preparation, and decision to submit the manuscript.

Medical writing assistance was provided by Shelley Batts, an employee of Analysis Group, Inc. Support for this assistance was funded by Takeda Development Center Americas, Inc.

All authors participated in the design of the study and contributed to the manuscript development. Data were analyzed by Analysis Group and interpreted in collaboration with all other authors. All named authors meet the International Committee of Medical Journal Editors (ICMJE) criteria for authorship for this manuscript, take responsibility for the integrity of the work as a whole, and have given final approval to the version to be published.

All authors had full access to all of the data in this study and take complete responsibility for the integrity of the data and accuracy of the data analysis.

Disclosures. Raafat Seifeldin is an employee of Takeda and owns stock/stock options. Paresh Chaudhari is a former employee of Takeda and owns stock/stock options. Zhou Zhou is an employee of Analysis Group, Inc., which has received consultancy fees from Takeda. Hongbo Yang is an employee of Analysis Group, Inc., which has received consultancy fees from Takeda. Jing Zhao is an employee of Analysis Group, Inc., which has received consultancy fees from Takeda. Anna P. Fang is an employee of Analysis Group, Inc., which has received consultancy fees from Takeda. Eric Q. Wu is an employee of Analysis Group, Inc., which has received consultancy fees from Takeda. Ernest H. Law was supported by a 2014-2016 UIC/ Takeda Fellowship in Health Economics and Outcomes Research. Ruixuan Jiang was 
supported by a 2015-2017 UIC/Takeda Fellowship in Health Economics and Outcomes Research.

Compliance with Ethics Guidelines. This article does not contain any new studies with human or animal subjects performed by any of the authors. All patient data were de-identified and complied with the patient confidentiality requirements of the Health Insurance Portability and Accountability Act.

Open Access. This article is distributed under the terms of the Creative Commons Attribution-NonCommercial 4.0 International License (http://creativecommons.org/licenses/ by-nc/4.0/), which permits any noncommercial use, distribution, and reproduction in any medium, provided you give appropriate credit to the original author(s) and the source, provide a link to the Creative Commons license, and indicate if changes were made.

\section{REFERENCES}

1. Schena FP, Gesualdo L. Pathogenetic mechanisms of diabetic nephropathy. J Am Soc Nephrol. 2005;16(Suppl 1):S30-3.

2. Tuttle KR, Bakris GL, Bilous RW, et al. Diabetic kidney disease: a report from an ADA Consensus Conference. Diabetes Care. 2014;37(10):2864-83.

3. Ahmad J. Management of diabetic nephropathy: recent progress and future perspective. Diabetes Metab Syndr. 2015;9(4):343-58.

4. de Boer IH, Rue TC, Hall YN, Heagerty PJ, Weiss NS, Himmelfarb J. Temporal trends in the prevalence of diabetic kidney disease in the United States. JAMA. 2011;305(24):2532-9.

5. Valmadrid CT, Klein R, Moss SE, Klein BE. The risk of cardiovascular disease mortality associated with microalbuminuria and gross proteinuria in persons with older-onset diabetes mellitus. Arch Intern Med. 2000;160(8):1093-100.

6. Kim JJ, Hwang BH, Choi IJ, et al. A prospective two-center study on the associations between microalbuminuria, coronary atherosclerosis and long-term clinical outcome in asymptomatic patients with type 2 diabetes mellitus: evaluation by coronary CT angiography. Int J Cardiovasc Imaging. 2015;31(1):193-203.

7. Gross JL, de Azevedo MJ, Silveiro SP, Canani LH, Caramori ML, Zelmanovitz T. Diabetic nephropathy: diagnosis, prevention, and treatment. Diabetes Care. $2005 ; 28(1): 164-76$.

8. The Professional Practice Committee of the ADA. American Diabetes Association (ADA) standards of medical care in diabetes-2015. Diabetes Care. 2015;38(Suppl 1):S1-S2.

9. Hajhosseiny R, Khavandi K, Jivraj N, Mashayekhi S, Goldsmith DJ, Malik RA. Have we reached the limits for the treatment of diabetic nephropathy? Expert Opin Investig Drugs. 2014;23(4):511-22.

10. Dhodi JB, Mestry SN, Juvekar AR. Diabetic nephropathy-genesis, prevention, and treatment. Int J Pharm Pharm Sci. 2014;6(9):42-7.

11. Vivian E, Mannebach C. Therapeutic approaches to slowing the progression of diabetic nephropathyis less best? Drugs in Context. 2013;2013:212249.

12. Vupputuri S, Kimes TM, Calloway MO, et al. The economic burden of progressive chronic kidney disease among patients with type 2 diabetes. J Diabetes Complicat. 2014;28(1):10-6.

13. Tuttle KR, Bakris GL, Bilous RW, et al. Diabetic kidney disease: a report from an ADA Consensus Conference. Am J Kidney Dis. 2014;64(4):510-33.

14. Ward A, Alvarez P, Vo L, Martin S. Direct medical costs of complications of diabetes in the United States: estimates for event-year and annual state costs (USD 2012). J Med Econ. 2014;17(3):176-83.

15. Slabaugh SL, Curtis BH, Clore G, Fu H, Schuster DP. Factors associated with increased healthcare costs in Medicare Advantage patients with type 2 diabetes enrolled in a large representative health insurance plan in the US. J Med Econ. 2015;18(2):106-12.

16. Gordois A, Scuffham P, Shearer A, Oglesby A. The health care costs of diabetic nephropathy in the United States and the United Kingdom. J Diabetes Complicat. 2004;18(1):18-26.

17. Yeaw J, Halinan S, Hines D, et al. Direct medical costs for complications among children and adults with diabetes in the US commercial payer setting. Appl Health Econ Health Policy. 2014;12(2):219-30.

18. Nichols GA, Vupputuri S, Lau H. Medical care costs associated with progression of diabetic nephropathy. Diabetes Care. 2011;34(11):2374-8. 
19. Adler AI, Stevens RJ, Manley SE, et al. Development and progression of nephropathy in type 2 diabetes: the United Kingdom prospective diabetes study (UKPDS 64). Kidney Int. 2003;63(1):225-32.

20. Quan H, Sundararajan V, Halfon P, et al. Coding algorithms for defining comorbidities in ICD-9-CM and ICD-10 administrative data. Med Care. 2005;43(11):1130-9.

21. Gordon K, Smyth BJ. Fitting Tweedie's compound Poisson model to insurance claims data: dispersion modelling. ASTIN Bull. 2002;32(1):143-57.

22. Hillege HL, Fidler V, Diercks GF, et al. Urinary albumin excretion predicts cardiovascular and noncardiovascular mortality in general population. Circulation. 2002;106(14):1777-82.

23. van der Velde M, Halbesma N, de Charro FT, et al. Screening for albuminuria identifies individuals at increased renal risk. J Am Soc Nephrol. 2009;20(4):852-62.

24. UK Prospective Diabetes Study (UKPDS). X. Urinary albumin excretion over 3 years in diet-treated type 2 (non-insulin-dependent) diabetic patients, and association with hypertension, hyperglycaemia and hypertriglyceridaemia. Diabetologia. 1993;36(10):1021-9. 